\title{
Molecular Mechanics of Cells and Tissues
}

\author{
JONATHON HOWARD \\ Max Planck Institute of Molecular Cell Biology and Genetics, Pfotenhauerstrasse 108, 01307 Dresden, Germany
}

(Received 22 January 2008; accepted 29 January 2008; published online 11 March 2008)

\begin{abstract}
In this review I address the question: can we understand the mechanics of organelles and cells based on the material properties and spatial arrangements of the cytoskeletal molecules such as microtubules, actin filaments and the host of associated proteins that crosslink them? In other words, can we do a structural analysis of a cell, as a mechanical engineer would perform a structural analysis on the design of a building or a bridge? This question can now be addressed because mechanical properties, such as stiffness and flexural rigidity, have been measured for several cytoskeletal proteins using single-molecule techniques. Owing to their small size, individual filaments are very soft and are readily bent by thermal and motor-driven forces. Strength can be increased by crosslinking. Crosslinked bundles are a first step in a hierarchy of structures formed with filaments. Recent work has clarified how the rigidity of crosslinked bundles varies with the length and the number of constituent filaments. From these considerations comes an appreciation for how the material properties of the cytoskeleton impose mechanical limitations on the design of organelles such as axonemes and hair bundles. The combination of theory and experiment promises to put cellular biomechanics on a firm molecular foundation.
\end{abstract}

Keywords-Cytoskeleton, Microtubule, Actin filament, Cross-linked bundle, Axoneme.

\section{INTRODUCTION}

When reading the classic textbook on biomechanics by Fung, ${ }^{15} \mathrm{I}$ am struck, as a cell biologist, by the paucity of molecules. There are terrific discussions of the mechanics of blood flow, blood cells, tendon, bone, and muscle, but little discussion of the mechanics of the molecules of the cytoskeleton-microtubules, actin filaments, intermediate filaments, and the host of associated proteins - that structure these cells and tissues. Even recent textbooks hardly mention molecules. ${ }^{10}$ In contrast, the cell biology textbooks (e.g., Refs. 1,57) have detailed descriptions of the cytoskeleton; but they do not take the crucial mechanical view

Address correspondence to Jonathon Howard, Max Planck Institute of Molecular Cell Biology and Genetics, Pfotenhauerstrasse 108, 01307 Dresden, Germany. Electronic mail: howard@mpi-cbg.de of the engineer who wants to know how the cytoskeleton shapes and stabilizes larger structures such as organelles, cells, and tissues. ${ }^{26}$ In this review I will sketch out a possible program in molecular biomechanics that might bridge the gap between molecules and cells.

\section{Recent Developments in Molecular and Cell Biology}

The traditional non-molecular view of biomechanics is changing rapidly because of two major developments over the last decades. The first is the sequencing of genomes. We now know all the putative proteins in organisms ranging from bacteria to man. Though most of these proteins are still not well enough characterized, we can often guess their function based on the domains they contain. The important thing is that the total number of proteins - the major building blocks of cells and tissues - though large, is finite. For biomechanics, this means that a molecular analysis of even very complex cellular structures such as the mitotic spindle that segregates the duplicated chromosomes in dividing cells and the leading edge of locomoting cells is a finite problem. For example, there are less than a 1000 genes essential for cell division in worms ${ }^{65}$ and about the same number associated with in the axoneme, the motile organelle of cilia and flagella. ${ }^{42}$ Even though the number of essential structural components is likely to be much smaller, the task of understanding the molecular basis for the structure of these organelles is still daunting.

The second major development is the detection and manipulation of single molecules. Assays were developed to reconstitute the movement of single motor proteins (kinesin) along cytoskeletal filaments (microtubules) using purified components. ${ }^{30} \mathrm{~A}$ variety of micromechanical techniques involving optical traps, ${ }^{13,67}$ glass fibers, ${ }^{49}$ and hydrodynamic loads ${ }^{32}$ could then be used to measure the forces generated by single motor proteins. At around the same time, the forces required to dissociate a single ligand molecule from a $\operatorname{protein}^{48,51}$ and to unfold a $\operatorname{protein}^{60}$ were 


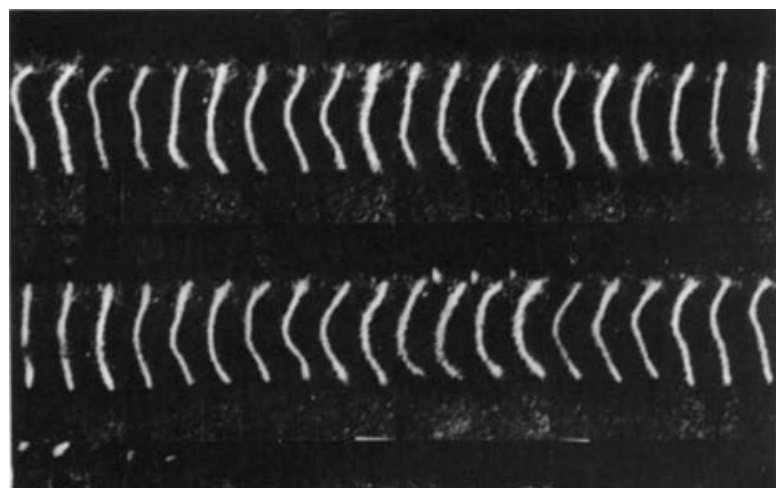

FIGURE 1. Thermal fluctuations of an actin filament. The filament is $11.5 \mu \mathrm{m}$ long and the sequential dark-field micrographs were taken at 1/12-s intervals. From Nagashima and Asakura. ${ }^{52}$

measured by atomic force microscopy. Total-internalreflection fluorescence microscopy allowed single fluorescently labeled proteins to be detected, ${ }^{14}$ and single-molecule fluorescence spectroscopy ${ }^{17}$ is now widely used in vitro and in cells. The first mechanical measurements on reconstituted cytoskeletal filaments were made using darkfield microscopy (Fig. 1): the stunning images of single actin filaments undergoing thermally induced fluctuations showed directly that these filaments are very soft - they are "semi-flexible," see below-and provided the first estimate of the flexural rigidity of cytoskeletal filaments. ${ }^{52}$ These observations ushered in a new era of single-molecule mechanics.

\section{Cellular and Molecular Biomechanics}

Application of single-molecule techniques has provided quite a detailed characterization of the mechanical properties of cytoskeletal proteins. The flexural rigidity, torsional rigidity, longitudinal stiffness, and tensile strength of actin filaments have been measured. ${ }^{71}$ The flexural rigidity of microtubules is known ${ }^{21}$ as is the stiffness of some of the proteins that crosslink the cytoskeletal filaments together, such as motor proteins ${ }^{73}$ nexin links in axonemes, ${ }^{43}$ spectrin that connects actin filaments together in a meshwork adjacent to the plasma membrane ${ }^{66}$ and ankyrin that links the actin-spectrin network to membrane proteins. ${ }^{40}$ Even though it is still early days in the characterization of the material properties of the cytoskeleton, there is enough known to raise the question that I want to focus on in this review: can we understand the mechanics of whole organelles and cells based on the mechanical properties and spatial arrangements of the cytoskeletal molecules? In other words, can we do a structural analysis of a cell, as a mechanical engineer would perform a structural analysis on the design of a building or a bridge? If we could, then this would put cellular biomechanics on a firm molecular foundation.

Why should we bother? I think that there are several reasons. First, it is intellectually satisfying to understand how beautiful organelles such as the axoneme, the mitotic spindle, and the hair bundle (the sensory organelle of hair cells in the inner ear) work as mechanical devices. Second, understanding the design principles underlying biological structures may provide inspiration or ideas for how to build new synthetic materials. Two notable examples are Velcro, that mimics a burr, and the anti-spoiling surfaces based on the nanostructure of the lotus leaf. ${ }^{53} \mathrm{~A}$ third reason is to understand the effects of physical trauma on cells, such as neurons. And lastly, when designing implants, it is possible that matching the mechanical impedances between inanimate materials and living tissues may be as important as chemical compatibility. This is because cells sense and respond to their mechanical environment (e.g., Ref. 4). For example, fibroblasts move away from surfaces with low rigidity ${ }^{44}$ and mesenchymal stem cells differentiate depending on the elasticity of the matrix in which they are cultured. ${ }^{12}$ The mechanical properties of cells and their environments are clearly very important for biological function.

\section{Design Principles}

Before we get into the nuts and bolts of the cytoskeleton, I will first contrast the design principles underlying cell architecture with those underlying everyday structures. Engineers design structures to be strong enough that they do not fall down. Beams are chosen with appropriate safety margins. An important consideration is the minimization of the cost of materials. The main problem is gravity: the strength of a column increases with its area but the weight increases with its volume. Consequently, as the height increases, the weight will eventually exceed the tensile strength of the material, and the structure will collapse. This sets the maximum size of buildings, trees, and elephants (see e.g., Ref. 24). On the other hand, gravity is not a problem for cells. As the overall dimension gets smaller, gravity becomes negligible. For example, a single motor protein (dimension $\sim 10 \mathrm{~nm}$ ) can generate enough force $(\sim 5 \mathrm{pN})$ to lift the weight of a red blood cell (dimension $\sim 10 \mu \mathrm{m}$ ) that is approximately 1000 times larger in linear dimension and a billions times larger in volume. One expects different design principles for different size scales.

Cells and organelles, like buildings and bridges, have structures that reflect or are a consequence of their function. For example, a bipolar mitotic spindle is a microtubule-based structure "designed" to 
segregate the duplicated chromosomes, and the hair bundle is "designed" to detect forces arising from sounds or body accelerations. Structure dictates function and vice versa in both the macroscopic and the microscope world. However, there are important differences. For cells, the design is arrived at through the trial and error process of evolution, rather than at the hand of the engineer. The construction is also very different. Buildings are constructed by architects and foremen who follow a plan and oversee the entire process. Cells have no master builder. Rather, cells are made of "smart bricks" that somehow know where they should be. This process of self-organization is very poorly understood and is a major open question in cell biology. I will come back to it at the end of this review.

Is there a single unifying concept underlying cell mechanics? One proposal is tensegrity. ${ }^{33}$ The idea is that the cell is a pre-stressed composite of tensile and compression elements, and the tension produces the integrity. The idea came from the sculptures of Kenneth Snelson (US Patent 3,169,611) and the term was coined by Buckminster Fuller. In the cell, the thin, cable-like actin filaments are postulated to be the tensile elements and the thick, pipe-like microtubules are the compression elements. This idea has some experimental support. For example, many cells are pre-stressed, and the depolymerization of the actin filaments can lead to cell elongation that depends on the microtubules ${ }^{38}$ or the extracellular matrix. ${ }^{62}$ However, other observations are not consistent with the tensegrity model. For example, actin filaments need not be in tension and microtubules need not be in compression. In the leading edge of locomoting cells, actin filaments push the membrane forward; ${ }^{58}$ so actin filaments can be in compression. In mitosis, shortening of microtubules pulls the chromosomes to the two poles ${ }^{55}$ and pulls the mitotic spindle towards the cortex; ${ }^{23}$ so microtubules can be in tension. Another weakness of the tensegrity concept is that it is a qualitative rather than quantitative one. How much tension? How strong? Surely detailed structural information is needed to determine whether a cell or organelle can withstand forces generated from within, by their motor proteins for example, or impinging from outside, from external pressure or shear.

\section{Mechanics of Cellular Materials}

To understand the mechanics of a cell or organelle one needs to consider the details of the arrangements of the cytoskeleton, just as a mechanical engineer needs to know the details of the plan and materials to know whether a building or bridge will hold up. But this is not a problem because electron microscopy can provide the information. Furthermore, dramatic improvements in electron cryo-microscopy have greatly improved the preservation of samples, and atomic structures can now be modeled into the EM images. This is beautifully demonstrated in recent images of the leading edge ${ }^{47}$ and the sperm flagellum. ${ }^{54}$ Thus it is becoming feasible to span from atomic dimensions $(0.1-1 \mathrm{~nm})$ to cellular dimensions (up to $1000 \mathrm{~nm}$ ). This gives additional impetus to the foundations of biomechanics: will it be possible one day to build an atomic model of a cell and predict its mechanical properties?

Given the arrangement of the filaments and the crossbridges between them, what are the mechanical properties of the structure? This seems like a straightforward exercise in mechanical engineering $101 .{ }^{18}$ But this is not the case. There are lots of surprises. When filaments are crosslinked together there is an interesting transition as one goes from a collection of independent rods whose strength increases only in proportion to the number of individuals, to a tightly coupled bundle whose strength increases with the square of the number. ${ }^{29}$ The latter is the proverbial strength in numbers. Pre-stress can also stiffen cellular structures by tautening the crosslinks between filaments. ${ }^{16}$ Cytoskeletal polymers are also interesting because, as mentioned earlier, they are soft enough to be significantly bent by thermal forces. This brings up a fundamental property of shape at the molecular level: shape is a mechanical property because it is contingent on the strength of the structure. And third, the cytoskeleton is highly dynamic - the filaments grow and shrink - and this means that the mechanical properties depend on the time-scale of the measurement. In the remainder of this review I will sketch out some of these ideas and then take stock of where we are, where we need to go in the future. An important conclusion is that there is still a lot to do!

\section{Bending and Buckling of Beams}

The building block of structures is the beam (Fig. 2). A beam is a slender rod whose cross-section is much smaller than its length $(L)$; we will make this statement more precise later on. If the beam is only slightly bent, so that the tangent angle is small everywhere, then we can represent its shape as a function $y(x)$ (Fig. 2a). If a force per unit length $f(x)$ acts on the beam and bends it, then the total energy of the beam depends on its shape according to

$$
U[y(x)] \approx \int_{0}^{L} \frac{1}{2} \kappa\left[\frac{d^{2} y}{d x^{2}}(x)\right]^{2} \cdot d x-\int_{0}^{L} f(x) y(x) \cdot d x
$$

$\kappa$ is the flexural rigidity (also known as the bending stiffness) and $d^{2} y / d x^{2}$ is approximately equal to the 
(a)

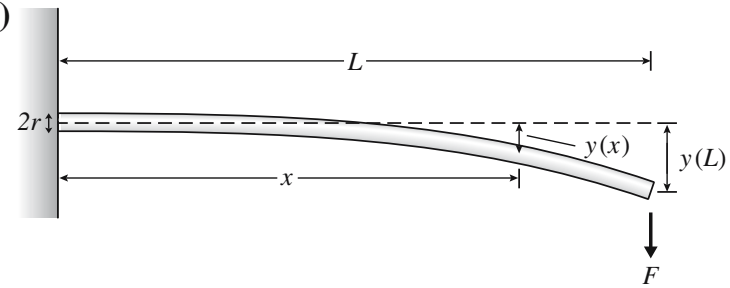

(b)

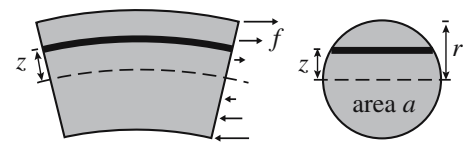

FIGURE 2. A bent beam. (a) The deformation of a cantilevered beam. (b) Left: the stresses and strains within a small longitudinal segment. As the beam bends material above the neutral plane (dashed) is stretched and material below is compressed. Right: the cross-section. The resistance to bending is proportional to the second moment of area about the neutral plane.

curvature. The first term represents the bending energy of the beam, and the second term is the work that the force does on it. For an isotropic and homogenous material, the flexural rigidity is $\kappa=E I$ where $E$ is the Young's modulus, that relates the stress in the material to its strain, and $I=\int z^{2} d a$ is the second moment of area (Fig. 2b). $I$ is proportional to the fourth power of the radius, $r$ ( $I=\pi r^{4} / 4$ for a circular section). At mechanical equilibrium, the beam will adopt a shape that minimizes its energy. From the calculus of variations, it can be shown that $y(x)$ satisfies the following differential equation

$$
\kappa \frac{d^{4} y}{d x^{4}}=f(x)
$$

In the case of a clamped beam $\left(y(0)=y^{\prime}(0)=0\right)$ with a concentrated force, $F$, at its free end, the deflection of the free end is $y(L)=F L^{3} / 3 \kappa$. Because the deflection is proportional to the force, we can think of this cantilevered beam as a spring with stiffness

$$
K=\frac{3 \kappa}{L^{3}}
$$

The flexural rigidity of microtubules, measured with optical tweezers, hydrodynamic forces and thermal forces, is in the range $10-40 \times 10^{-24} \mathrm{~N} \mathrm{~m}^{2}$ (Refs. 21,36,50). Using the cross-section of the microtubule derived from structural studies ${ }^{41}$ to estimate the second moment of area, and assuming that the protein material is isotropic and homogenous, we obtain a Young's modulus $E \approx 2 \mathrm{GPa}$. This is similar to the Young's modulus of hard plastics, ${ }^{35}$ dry hair, hoofs, and tendons. ${ }^{75}$ The flexural rigidity of actin filaments is much less than that of microtubules. However, given that the cross-sectional area of an actin filament is much smaller than that of the microtubule $\left(20 \mathrm{~nm}^{2}\right.$ compared to $200 \mathrm{~nm}^{2}$ ), the Young's modulus is similar. Thus the differences in mechanical properties of actin and microtubules can primarily be attributed to the different geometries of the filaments rather than their material properties. This is interesting because the constituent proteins-actin and tubulin-have no structural similarity. Several other filamentous proteins have similar material properties. ${ }^{26}$ This suggests that isotropy and homogeneity may be reasonable approximations, at least in the case of structural proteins. An important future direction will be to understand the material properties of proteins in terms of their molecular structure. $2,5,66$

The flexural rigidity of a filament determines its resistance to a compressive load. A central result in mechanical engineering textbooks, is the existence of a maximum compressive load that a beam can withstand before it buckles. The critical force is

$$
F_{\mathrm{c}}=\pi^{2} \frac{\kappa}{L^{2}}
$$

(assuming the ends are fixed but free to swivel). Because the flexural rigidity scales with 4th power of the diameter, the buckling force decreases quickly as the overall dimensions of a beam are scaled down. A single motor protein that generates a force of $\sim 6 \mathrm{pN}$ can buckle a $10-\mu \mathrm{m}$-long microtubule. ${ }^{20}$ A single myosin molecule can buckle an actin filament that is only $\sim 1 \mu \mathrm{m}$ long.

\section{Cytoskeletal Filaments are Semiflexible Polymers}

Owing to their small diameter, cytoskeletal filaments are so flexible that they are appreciably bent by thermal forces. The spring constant of a cantilevered microtubule of length $10 \mu \mathrm{m}$ is only $0.1 \mu \mathrm{N} / \mathrm{m}$. This is very soft: a force of only $0.1 \mathrm{pN}$ at the end will deflect it through $1 \mu \mathrm{m}$. The amount of thermally induced bending can be estimated using the equipartition theorem: the average potential energy equals $\frac{1}{2} k_{\mathrm{B}} T$ where $k_{\mathrm{B}}$ is the Boltzmann constant and $T$ is absolute temperature. The potential energy of a cantilevered spring deflected through a distance $x$ is $\frac{1}{2} K x^{2}$, and so for the a $10 \mu \mathrm{m}$-long microtubule the standard deviation of the end deflection at room temperature is $\approx 0.25 \mu \mathrm{m}$, readily observable under an optical microscope. The thermal fluctuations of actin filaments are even more pronounced (Fig. 1), because of their lower rigidity.

In describing the thermally induced bending of a filament, the persistence length, $L_{\mathrm{p}}$ is a useful concept. It is 
proportional to the flexural rigidity $\left(L_{\mathrm{p}}=\kappa / k_{\mathrm{B}} T\right)$ and corresponds to the length over which the angle at one end becomes uncorrelated with the angle at the other (see Ref. 27 for a rigorous definition). The persistence lengths of microtubules, actin filaments and DNA are respectively $6 \mathrm{~mm}, 10 \mu \mathrm{m}$, and $50 \mathrm{~nm}$. Thus actin filaments are semiflexible over cellular dimensions, while microtubules are stiff and DNA is very flexible.

\section{Filament Bundles}

In order to build rigid structures, the filaments must be crosslinked together. Consider two filaments coupled by shear springs of stiffness $k$ per unit length that resist sliding between the filaments. There are two extreme cases (Fig. 3). If the shear springs are relatively soft, the filaments will not change length during bending. The two filaments will bend independently (Fig. 3a), and the flexural rigidity will simply be twice that of the individual filament, $2 \kappa$. On the other hand, if the shear springs are very rigid so that no sliding is possible, then the flexural rigidity will be much larger. This is because in order to bend, one filament has to be stretched while the other compressed (Fig. 3b). If the separation is much larger than the diameters of the individual filaments, then the flexural rigidity will be $2 E a R^{2}$ where $a$ is the cross-sectional area of each filament and $2 R$ is the center-to-center separation. Clearly strongly-crosslinked filaments will be much more resistant to both bending and buckling.

The strength of coupling between the filaments determines how the rigidity scales with the number of filaments. If the coupling is weak, then the flexural rigidity of a symmetric array is proportional to the number of constituent filaments, $N$. If the coupling is strong then it scales as $N^{2}$ (Refs. 3,29). This scaling behavior has been reconstituted with purified actin filaments and the crosslinking protein fascin: at low crosslinker density the flexural rigidity of the bundle scales with $N$ and at high density with $N^{2}$ (Ref. 9). The stiffness of a hair bundle increases in proportion to the number of its stereocilia, the enlarged actin-filament-containing microvilli that form the organelle. This indicates that the links between stereocilia that oppose shear are relatively soft. ${ }^{29}$ By contrast, the stereocilia themselves behave as rigid rods that pivot about their insertions into the apical surface of the cell, ${ }^{29}$ indicating that the individual actin filaments within the stereocilia are strongly coupled by the crosslinking proteins fimbrin and/or espin. Because the fimbrin-related protein plastin is a weak crosslinker, ${ }^{9}$ the strong coupling may be mediated by espin, mutations in whose gene leads to deafness in mice and humans. ${ }^{63}$

Recent work has shown that the crossover between weak and strong coupling depends on the length of the filament. For a pair of crosslinked filaments, the critical length is 3,25

$$
L_{\mathrm{c}}^{2}=\frac{\pi^{2}}{8} \frac{E a}{k} .
$$

Above this length coupling is strong. The reason for the length dependence is that the total resistance to shear gets larger as the filament gets longer (the shear springs are in parallel). An interesting corollary of the length-dependent crossover, is that the flexural rigidity of a bundle is expected to depend on the length. Such a length-dependent flexural rigidity, attributed to weak crosslinking, has been observed for crosslinked bundles of microtubules in pillar cells in the inner ear. ${ }^{70}$ Even single microtubules have been reported to have a length-dependent flexural rigidity. ${ }^{37,39,56}$ Microtubules can therefore be viewed as a bundle of 13 protofilaments that are quite weakly crosslinked together and therefore mechanically anisotropic. The latter study (Ref. 56) found that the flexural rigidity of microtubules increased 10-fold with length, with the halfmaximum rigidity occurring at a length of $\sim 20 \mu \mathrm{m}$. Because the shear stiffness is related to the shear modulus by $G=k R / r$ (Fig. 4) and because the crossover length is $1000-2000$ times greater than the diameters of the microtubule and the protofilament, the ratio of the shear modulus to the Young's modulus is predicted to be very small, $\sim 10^{-6}$. For comparison, an (a)

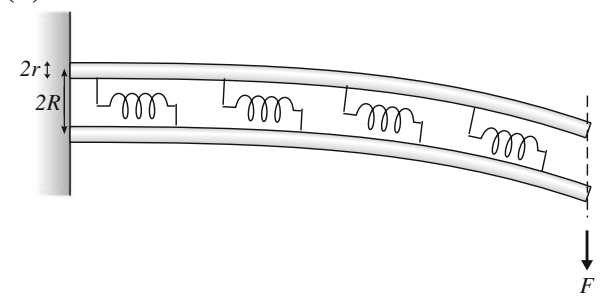

(b)

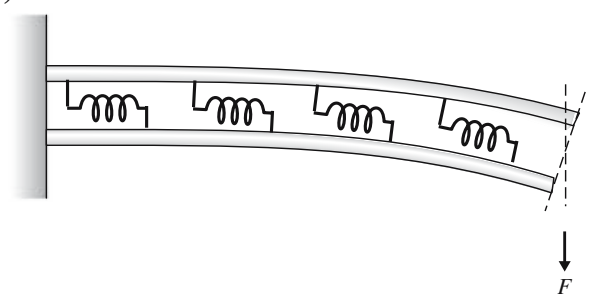

FIGURE 3. A pair of crosslinked filaments. (a) When the shear springs are soft, the filaments bend but remain the same length. (b) When the springs are rigid, bending can only be accomplished by stretching the upper filament and compressing the lower one. The over flexural rigidity is larger in the latter case. 


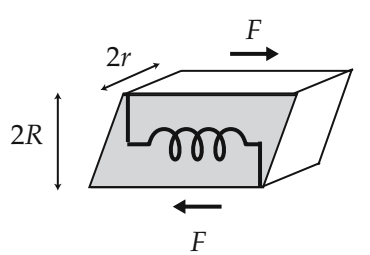

FIGURE 4. A spring that opposes shear can be equated with the shear modulus (see text).

isotropic and homogenous material has a ratio equal to $1 /(2(1+\sigma)) \approx 1 / 2.5$ where $\sigma$ is the Poisson ratio, which for solids has typical values of $\sim 0.25$. Though molecular simulations do indicate that the bonds between tubulins are weaker between protofilaments than within protofilaments, ${ }^{64}$ the extent of the anisotropy is surprising given the atomic model of the microtubule has "a fair degree of complementarity across the interface" between protofilaments. ${ }^{41}$ It will be important to confirm the mechanical measurements as well as to model the mechanical properties of the inter-protofilament connections.

The length-dependent crossover has interesting implications for the design of axonemes (Fig. 5). Axonemes beat due to the sliding between the doublet microtubules driven by the motor protein dynein. Recent work from our lab has shown that a model in which load accelerates the detachment of the dyneins from the microtubule can account for the propagation of the beat. ${ }^{59}$ However, the model will break down if there is no sliding and therefore the wavelength of the beat, $\lambda$, cannot be too long. The critical wavelength is $\lambda_{\mathrm{c}}^{2}=2 \pi^{2} E a / k$. For parameters measured for the bull sperm, ${ }^{58}$ where the shear stiffness is contributed primarily by the motors themselves, the critical wavelength is $\sim 70 \mu \mathrm{m}$. Interestingly, the wavelength of the flagellar beat of bull sperm is $\sim 50 \mu \mathrm{m}$, and a survey of beat wavelengths from a broad variety of species from sea urchins to humans indicates that all are less than $70 \mu \mathrm{m}$ (Ref. 7). These arguments suggest that the axonemal beat is constrained by the material properties of the constituent molecules. To what extent are other cellular structures limited by the material properties of their constituent molecules? What are the safety factors?

\section{Future Prospects}

We are just beginning to get an understanding of how molecules shape cells. Gravity is not a limitation because inertial forces are very small at molecular and cellular dimensions. However, as we scale down beams, the flexural rigidity becomes very small because the second moment of area scales with the 4th power of the diameter. This has several implications. First, filaments bend under thermal forces, and this puts an upper limit on the lengths of individual filaments as structural elements. Second, filaments buckle more easily and this sets an upper limit of the length of a filament as a compressive element. In this regard it is interesting that the viscoelastic properties of the cytoplasm, probably due to the presence of numerous actin filaments, can oppose the formation of large amplitude buckles and this can significantly strengthen microtubules as compression elements. ${ }^{6}$ And third, in order to build strong structures that resist bending and buckling, the filaments can be strengthened by crosslinking. Bundling represents a second step in a hierarchy of possible structures formed with filaments. Interestingly the flexural rigidity of a bundle depends on its length and number of constituent filaments. Full coupling is only achieved when the bundle is long enough, in which case it behaves as a "slender filament" $\left(L^{2} \gg N E a / k \approx E A / G\right.$ where $A$ is the cross-section area of the bundle). ${ }^{3}$ At much shorter lengths, shear between filaments occurs, and this is useful in structures such as axonemes and hair bundles where shear plays a motor or sensory role. But the length dependence sets an upper size of these structures, else shear becomes secondary to filament stretch and compression.

We have not considered dynamics of the cytoskeleton: through depolymerization and polymerization

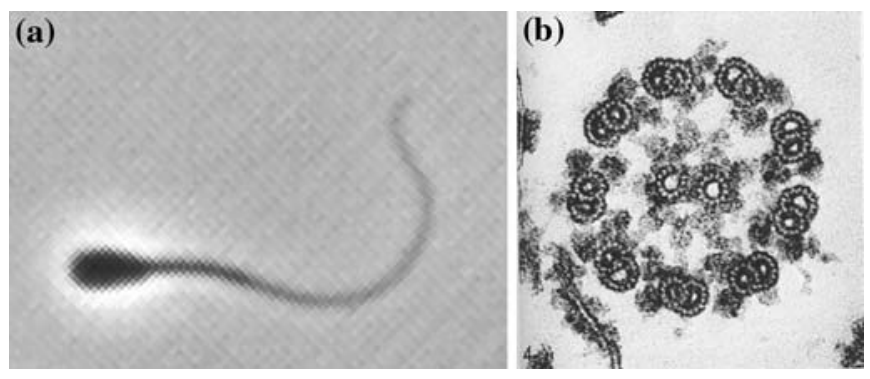

FIGURE 5. Sperm. (a) A bull sperm with a $57-\mu \mathrm{m}$-long tail. From Riedel-Kruse et al. $^{59}$ (b) Electron micrograph of the cross-section through a sea-urchin sperm showing the nine doublet microtubules surrounding the central pair of singlet microtubules. The density between doublets corresponds to the dynein motors. The diameter of the complete microtubules is $25 \mathrm{~nm}$. From Tilney et al. 69 
the cytoskeletal filaments can turn over in as little as $1 \mathrm{~min}$ in some cells. This turnover is crucial in cell motility and division. An interesting consequence of this turnover is that the stiffness of the cytoskeletal network will depend on the time-scale, with high stiffness at short timescales and low stiffness on long timescales. ${ }^{28}$ The binding and unbinding of crosslinks will also lead to lower stiffness on long timescales. ${ }^{74}$ Furthermore, thermal fluctuations of semi-flexible filaments within gels also lead to interesting viscoelastic properties. ${ }^{19,34}$ Thus there are several mechanisms by which the dynamical properties of the cytoskeleton can influence the rheology of gels and cells.

The lengths of filaments determine the size and strength of cellular structures. But how is length regulated? ${ }^{45}$ In muscle and RNA viruses, long molecules - titin in the case of muscle and the RNA in the case of the virus - act as molecular rulers to template length. However, cytoskeletal filaments are dynamic polymers and the length must somehow reflect the balance between growth and shrinkage. In this case, length control is difficult because polymers grow and shrink at their ends and there is no obvious connection of these processes to the length of the polymer. One possibility is that length may be regulated by the sliding force between polymers. ${ }^{22}$ Another possibility is the action of depolymerizing kinesins such as Kip3p that depolymerizes long microtubules faster than short ones. ${ }^{72}$ In combination with length-independent growth, this would give length control. ${ }^{31}$ The identification of mutations in cilia length in the alga Chlamydomonas is an exciting approach towards understanding the molecular basis of length control. ${ }^{68}$ Perhaps length feedback through length-sensing motors and signaling networks plays the role of "master builder" in setting the size of cellular structures.

This review has focused on the cytoskeletal filaments due to the research interests of the author. However, mechanics plays crucial roles in many other cellular processes. To properly understand the cell, we will need to know the mechanical properties of the chromatin, ${ }^{8,76}$ the nuclear envelope $\mathrm{e}^{11,61}$ and the myriad of membraneassociated proteins that shape membranes and play essential roles in endocytosis and exocytosis. ${ }^{46,77}$

\section{ACKNOWLEDGMENTS}

The author thanks Drs M. Bathe and C.P. Brangwynne for stimulating discussions and Drs D. Odde and M. Zanic for comments on an earlier draft of the manuscript. Original research by the author has been supported by the Max Planck Society and the National Institutes of Health.

\section{OPEN ACCESS}

This article is distributed under the terms of the Creative Commons Attribution Noncommercial License which permits any noncommercial use, distribution, and reproduction in any medium, provided the original author(s) and source are credited.

\section{REFERENCES}

${ }^{1}$ Alberts, B., A. Johnson, J. Lewis, M. Raff, K. Roberts, and P. Walter. Molecular Biology of the Cell. New York: Garland Pub, 2002.

${ }^{2}$ Bathe, M. A finite element framework for computation of protein normal modes and mechanical response. Proteins 70:1595-1609, 2007.

${ }^{3}$ Bathe, M., C. Heussinger, M. M. Claessens, A. Bausch, and E. Frey. Cytoskeletal bundle mechanics. Biophys. J., 2008 (in press).

${ }^{4}$ Bershadsky, A. D., N. Q. Balaban, and B. Geiger. Adhesion-dependent cell mechanosensitivity. Annu. Rev. Cell Dev. Biol. 19:677-695, 2003.

${ }^{5}$ Birmanns, S., and W. Wriggers. Multi-resolution anchorpoint registration of biomolecular assemblies and their components. J. Struct. Biol. 157(1):271-280, 2007.

${ }^{6}$ Brangwynne, C. P., F. C. MacKintosh, S. Kumar, N. A. Geisse, J. Talbot, L. Mahadevan, K. K. Parker, D. E. Ingber, and D. A. Weitz. Microtubules can bear enhanced compressive loads in living cells because of lateral reinforcement. J. Cell Biol. 173(5):733-741, 2006.

${ }^{7}$ Brennen, C., and H. Winet. Fluid mechanics of propulsion by cilia and flagella. Ann. Rev. Fluid Mech. 9:339-398, 1977.

${ }^{8}$ Bustamante, C., Z. Bryant, and S. B. Smith. Ten years of tension: single-molecule DNA mechanics. Nature 421(6921):423-427, 2003.

${ }^{9}$ Claessens, M. M., M. Bathe, E. Frey, and A. R. Bausch. Actin-binding proteins sensitively mediate F-actin bundle stiffness. Nat. Mater. 5(9):748-753, 2006.

${ }^{10}$ Cowin, S. C., and S. B. Doty. Tissue Mechanics. New York: Springer, 2007.

${ }^{11}$ Dahl, K. N., S. M. Kahn, K. L. Wilson, and D. E. Discher. The nuclear envelope lamina network has elasticity and a compressibility limit suggestive of a molecular shock absorber. J. Cell Sci. 117(Pt 20):4779-4786, 2004.

${ }^{12}$ Engler, A. J., S. Sen, H. L. Sweeney, and D. E. Discher. Matrix elasticity directs stem cell lineage specification. Cell 126(4):677-689, 2006.

${ }^{13}$ Finer, J. T., R. M. Simmons, and J. A. Spudich. Single myosin molecule mechanics: piconewton forces and nanometre steps [see comments]. Nature 368(6467):113-119, 1994.

${ }^{14}$ Funatsu, T., Y. Harada, M. Tokunaga, K. Saito, and T. Yanagida. Imaging of single fluorescent molecules and individual ATP turnovers by single myosin molecules in aqueous solution [see comments]. Nature 374(6522):555$559,1995$.

${ }^{15}$ Fung, Y. C. Biomechanics: Mechanical Properties of Living Tissues. New York: Springer-Verlag, 1993.

${ }^{16}$ Gardel, M. L., F. Nakamura, J. Hartwig, J. C. Crocker, T. P. Stossel, and D. A. Weitz. Stress-dependent elasticity of composite actin networks as a model for cell behavior. Phys. Rev. Lett. 96(8):088102, 2006. 
${ }^{17}$ Gell, C., D. Brockwell, and A. Smith. Handbook of Single Molecule Fluorescence Spectroscopy. Oxford, New York: Oxford University Press, 2006.

${ }^{18}$ Gere, J. M. Mechanics of Materials. Belmont, CA: Brooks/Cole-Thomas Learning, 2004.

${ }^{19}$ Gittes, F., and F. C. MacKintosh. Dynamic shear modulus of a semiflexible polymer network. Phys. Rev. E 58:12411244, 1998.

${ }^{20}$ Gittes, F., E. Meyhöfer, S. Baek, and J. Howard. Directional loading of the kinesin motor molecule as it buckles a microtubule. Biophys. J. 70:418-429, 1996.

${ }^{21}$ Gittes, F., B. Mickey, J. Nettleton, and J. Howard. Flexural rigidity of microtubules and actin filaments measured from thermal fluctuations in shape. J. Cell Biol. 120(4):923934, 1993.

${ }^{22}$ Goshima, G., R. Wollman, N. Stuurman, J. M. Scholey, and R. D. Vale. Length control of the metaphase spindle. Curr. Biol. 15(22):1979-1988, 2005.

${ }^{23}$ Grill, S. W., P. Gonczy, E. H. Stelzer, and A. A. Hyman. Polarity controls forces governing asymmetric spindle positioning in the Caenorhabditis elegans embryo. Nature 409(6820):630-633, 2001.

${ }^{24}$ Haldane, J. B. S. On Being the Right Size. Oxford University Press, 1985.

${ }^{25}$ Heussinger, C., M. Bathe, and E. Frey. Statistical mechanics of semiflexible bundles of wormlike polymer chains. Phys. Rev. Lett. 99(4):048101, 2007.

${ }^{26}$ Howard, J. Mechanics of Motor Proteins and the Cytoskeleton. Sunderland, MA: Sinauer Associates, 2001.

${ }^{27}$ Howard, J. Mechanics of motor proteins. In: Physics of Biomolecules and Cells, edited by H. Flyvbjerg, F. Jülicher, P. Ormos, and F. David. Berlin: Springer-Verlag, 2002.

${ }^{28}$ Howard, J. Elastic and damping forces generated by confined arrays of dynamic microtubules. Phys. Biol. 3(1):54 66, 2006.

${ }^{29}$ Howard, J., and J. F. Ashmore. Stiffness of sensory hair bundles in the sacculus of the frog. Hear Res. 23(1):93-104, 1986.

${ }^{30}$ Howard, J., A. J. Hudspeth, and R. D. Vale. Movement of microtubules by single kinesin molecules. Nature 342(6246):154-158, 1989.

${ }^{31}$ Howard, J., and A. A. Hyman. Microtubule polymerases and depolymerases. Curr. Opin. Cell Biol. 19(1):31-35, 2007.

${ }^{32}$ Hunt, A. J., F. Gittes, and J. Howard. The force exerted by a single kinesin molecule against a viscous load. Biophys. $J$. 67(2):766-781, 1994.

${ }^{33}$ Ingber, D. E. Tensegrity: the architectural basis of cellular mechanotransduction. Annu. Rev. Physiol. 59:575-599, 1997.

${ }^{34}$ Janmey, P. A., and D. A. Weitz. Dealing with mechanics: mechanisms of force transduction in cells. Trends Biochem. Sci. 29(7):364-370, 2004.

${ }^{35}$ Kaye, G. W. C., and T. H. Laby. Tables of Physical and Chemical Constants and Some Mathematical Functions. London, New York: Longman, 1986.

${ }^{36}$ Kikumoto, M., M. Kurachi, V. Tosa, and H. Tashiro. Flexural rigidity of individual microtubules measured by a buckling force with optical traps. Biophys. J. 90:1687-1696, 2006.

${ }^{37}$ Kis, A., S. Kasas, B. Babic, A. J. Kulik, W. Benoit, G. A. Briggs, C. Schonenberger, S. Catsicas, and L. Forro. Nanomechanics of microtubules. Phys. Rev. Lett. 89(24): 248101, 2002.

${ }^{38}$ Kolodney, M. S., and E. L. Elson. Contraction due to microtubule disruption is associated with increased phosphorylation of myosin regulatory light chain. Proc. Natl. Acad. Sci. USA 92(22):10252-10256, 1995.

${ }^{39}$ Kurachi, M., M. Hoshi, and H. Tashiro. Buckling of a single microtubule by optical trapping forces: direct measurement of microtubule rigidity. Cell Motil. Cytoskeleton 30(3):221-228, 1995.

${ }^{40}$ Lee, G., K. Abdi, Y. Jiang, P. Michaely, V. Bennett, and P. E. Marszalek. Nanospring behaviour of ankyrin repeats. Nature 440(7081):246-249, 2006.

${ }^{41}$ Li, H., D. J. DeRosier, W. V. Nicholson, E. Nogales, and K. H. Downing. Microtubule structure at 8 A resolution. Structure 10(10):1317-1328, 2002.

${ }^{42} \mathrm{Li}$, J. B., J. M. Gerdes, C. J. Haycraft, Y. Fan, T. M. Teslovich, H. May-Simera, H. Li, O. E. Blacque, L. Li, C. C. Leitch, R. A. Lewis, J. S. Green, P. S. Parfrey, M. R. Leroux, W. S. Davidson, P. L. Beales, L. M. GuayWoodford, B. K. Yoder, G. D. Stormo, N. Katsanis, and S. K. Dutcher. Comparative genomics identifies a flagellar and basal body proteome that includes the BBS5 human disease gene. Cell 117(4):541-552, 2004.

${ }^{43}$ Lindemann, C. B., L. J. Macauley, and K. A. Lesich. The counterbend phenomenon in dynein-disabled rat sperm flagella and what it reveals about the interdoublet elasticity. Biophys. J. 89(2):1165-1174, 2005.

${ }^{44}$ Lo, C. M., H. B. Wang, M. Dembo, and Y. L. Wang. Cell movement is guided by the rigidity of the substrate. Biophys. J. 79(1):144-152, 2000.

${ }^{45}$ Marshall, W. F. Cellular length control systems. Annu. Rev. Cell Dev. Biol. 20:677-693, 2004.

${ }^{46} \mathrm{McMahon}, \mathrm{H}$. T., and J. L. Gallop. Membrane curvature and mechanisms of dynamic cell membrane remodelling. Nature 438(7068):590-596, 2005.

${ }^{47}$ Medalia, O., I. Weber, A. S. Frangakis, D. Nicastro, G. Gerisch, and W. Baumeister. Macromolecular architecture in eukaryotic cells visualized by cryoelectron tomography. Science 298(5596):1209-1213, 2002.

${ }^{48}$ Merkel, R., P. Nassoy, A. Leung, K. Ritchie, and E. Evans. Energy landscapes of receptor-ligand bonds explored with dynamic force spectroscopy. Nature 397(6714):50-53, 1999.

${ }^{49}$ Meyhoefer, E., and J. Howard. The force generated by a single kinesin molecule against an elastic load. Proc. Natl. Acad. Sci. USA 92(2):574-578, 1995.

${ }^{50}$ Mickey, B., and J. Howard. Rigidity of microtubules is increased by stabilizing agents. J. Cell Biol. 130(4):909-917, 1995.

${ }^{51}$ Moy, V. T., E. L. Florin, and H. E. Gaub. Intermolecular forces and energies between ligands and receptors. Science 266(5183):257-259, 1994.

${ }^{52}$ Nagashima, H., and S. Asakura. Dark-field light microscopic study of the flexibility of F-actin complexes. J. Mol. Biol 136(2):169-182, 1980.

${ }^{53}$ Neinhuis, C., and W. Barthlott. The purity of sacred lotus or escape from contamination in biological surfaces. Planta 202:1-8, 1997.

${ }^{54}$ Nicastro, D., J. R. McIntosh, and W. Baumeister. 3D structure of eukaryotic flagella in a quiescent state revealed by cryo-electron tomography. Proc. Natl. Acad. Sci. USA 102(44):15889-15894, 2005.

${ }^{55}$ Nicklas, R. B. Measurements of the force produced by the mitotic spindle in anaphase. J. Cell Biol. 97(2):542-548, 1983.

${ }^{56}$ Pampaloni, F., G. Lattanzi, A. Jonas, T. Surrey, E. Frey, and E. L. Florin. Thermal fluctuations of grafted microtubules provide evidence of a length-dependent persistence 
length. Proc. Natl. Acad. Sci. USA 103(27):10248-10253, 2006.

${ }^{57}$ Pollard, T. D., W. C. Earnshaw, and J. Lippincott-Schwartz. Cell Biology. Philadelphia: Saunders/Elsevier, 2008.

${ }^{58}$ Prass, M., K. Jacobson, A. Mogilner, and M. Radmacher. Direct measurement of the lamellipodial protrusive force in a migrating cell. J. Cell Biol. 174(6):767-772, 2006.

${ }^{59}$ Riedel-Kruse, I. H., A. Hilfinger, J. Howard, and F. Julicher. How molecular motors shape the flagellar beat. HFSP J 1:192-208, 2007.

${ }^{60}$ Rief, M., M. Gautel, F. Oesterhelt, J. M. Fernandez, and H. E. Gaub. Reversible unfolding of individual titin immunoglobulin domains by AFM. Science 276(5315): 1109-1112, 1997.

${ }^{61}$ Rowat, A. C., L. J. Foster, M. M. Nielsen, M. Weiss, and J. H. Ipsen. Characterization of the elastic properties of the nuclear envelope. J. R. Soc. Interface 2(2):63-69, 2005.

${ }^{62}$ Sawhney, R. K., and J. Howard. Molecular dissection of the fibroblast-traction machinery. Cell Motil. Cytoskeleton 58(3):175-185, 2004

${ }^{63}$ Sekerkova, G., L. Zheng, P. A. Loomis, E. Mugnaini, and J. R. Bartles. Espins and the actin cytoskeleton of hair cell stereocilia and sensory cell microvilli. Cell Mol. Life Sci. 63(19-20):2329-2341, 2006.

${ }^{64}$ Sept, D., N. A. Baker, and J. A. McCammon. The physical basis of microtubule structure and stability. Protein Sci. 12(10):2257-2261, 2003.

${ }^{65}$ Sonnichsen, B., L. B. Koski, A. Walsh, P. Marschall, B. Neumann, M. Brehm, A. M. Alleaume, J. Artelt, P. Bettencourt, E. Cassin, M. Hewitson, C. Holz, M. Khan, S. Lazik, C. Martin, B. Nitzsche, M. Ruer, J. Stamford, M. Winzi, R. Heinkel, M. Roder, J. Finell, H. Hantsch, S. J. Jones, M. Jones, F. Piano, K. C. Gunsalus, K. Oegema, P. Gonczy, A. Coulson, A. A. Hyman, and C. J. Echeverri. Full-genome RNAi profiling of early embryogenesis in Caenorhabditis elegans. Nature 434(7032):462-469, 2005.

${ }^{66}$ Sotomayor, M., and K. Schulten. Single-molecule experiments in vitro and in silico. Science 316(5828):1144-1148, 2007.
${ }^{67}$ Svoboda, K., and S. M. Block. Force and velocity measured for single kinesin molecules. Cell 77(5):773-784, 1994.

${ }^{68}$ Tam, L. W., N. F. Wilson, and P. A. Lefebvre. A CDKrelated kinase regulates the length and assembly of flagella in Chlamydomonas. J. Cell Biol. 176(6):819-829, 2007.

${ }^{69}$ Tilney, L. G., J. Bryan, D. J. Bush, K. Fujiwara, M. S. Mooseker, D. B. Murphy, and D. H. Snyder. Microtubules: evidence for 13 protofilaments. J. Cell Biol. 59(2 Pt 1): 267-275, 1973.

${ }^{70}$ Tolomeo, J. A., and M. C. Holley. Mechanics of microtubule bundles in pillar cells from the inner ear. Biophys. $J$. 73(4):2241-2247, 1997.

${ }^{71}$ Tsuda, Y., H. Yasutake, A. Ishijima, and T. Yanagida. Torsional rigidity of single actin filaments and actin-actin bond breaking force under torsion measured directly by in vitro micromanipulation. Proc. Natl. Acad. Sci. USA 93(23):12937-12942, 1996.

${ }^{72}$ Varga, V., J. Helenius, K. Tanaka, A. A. Hyman, T. U. Tanaka, and J. Howard. Yeast kinesin-8 depolymerizes microtubules in a length-dependent manner. Nat. Cell Biol. 8(9):957-962, 2006.

${ }^{73}$ Veigel, C., M. L. Bartoo, D. C. White, J. C. Sparrow, and J. E. Molloy. The stiffness of rabbit skeletal actomyosin cross-bridges determined with an optical tweezers transducer. Biophys. J. 75(3):1424-1438, 1998.

${ }^{74}$ Wachsstock, D. H., W. H. Schwarz, and T. D. Pollard. Cross-linker dynamics determine the mechanical properties of actin gels. Biophys. J. 66(3 Pt 1):801-809, 1994.

${ }^{75}$ Wainwright, S. A., W. D. Biggs, J. D. Currey, and J. M. Gosline. Mechanical Design in Organisms. Princeton, NJ: Princeton University Press, 1976.

${ }^{76}$ Yan, J., T. J. Maresca, D. Skoko, C. D. Adams, B. Xiao, M. O. Christensen, R. Heald, and J. F. Marko. Micromanipulation studies of chromatin fibers in Xenopus egg extracts reveal ATP-dependent chromatin assembly dynamics. Mol. Biol. Cell 18(2):464-474, 2007.

${ }^{77}$ Zimmerberg, J., and M. M. Kozlov. How proteins produce cellular membrane curvature. Nat. Rev. Mol. Cell Biol. 7(1):9-19, 2006. 